DOI: $10.3901 / J M E .2019 .13 .185$

\title{
微细钻头螺旋槽刀磨试验研究*
}

\author{
梁志强 ${ }^{1}$ 郭海新 $^{2}$ 张素燕 $^{2}$ 王西彬 $^{1}$ 周天丰 ${ }^{1}$ 刘志兵 ${ }^{1}$ \\ 李 玉 $^{3}$ 陈建军 ${ }^{3}$
}

(1. 北京理工大学先进加工技术国防重点学科实验室 北京 100081 ;

2. 北京理工大学机械与车辆学院 北京 100081 ;

3. 北方通用动力集团有限公司 大同 037036)

\begin{abstract}
摘要: 螺旋槽几何结构是影响钻头切削性能和微孔加工质量的重要因素。螺旋槽形状主要取决于刃磨参数、砂轮形状参数以 及砂轮位置参数, 通过调整砂轮的形状与位置参数即可以获得所需的螺旋槽形状。通过分析砂轮和螺旋槽之间的相对运动关 系, 建立微细钻头螺旋槽型的刃磨数学模型, 并基于 Matlab 软件对螺旋槽刃磨截形进行了数值仿真, 分析了砂轮形状与位置 参数对螺旋槽形状、径向前角和螺旋槽宽度的影响规律。研究结果表明砂轮边缘宽度、砂轮锥角、砂轮偏置角度和砂轮偏移 距离对螺旋槽截形有明显的影响。随着砂轮边缘宽度和砂轮锥角的增大, 径向前角没有变化, 但是螺旋槽宽度增大, 容屑空 间明显增大; 随着砂轮偏置角度的增大, 螺旋槽径向前角明显增大, 螺旋槽宽度减小; 随着砂轮偏移距离的增大, 径向前角 变化较小, 而螺旋槽宽度明显增大。基于所建立的刃磨数学模型, 刃磨出不同螺旋槽型微细钻头, 证实刃磨结果与仿真结果 具有较好的一致性。采用所制备的微细钻头进行钻削试验, 验证了螺旋槽结构对容屑与排屑能力具有重要影响。
\end{abstract}

关键词: 螺旋槽形状; 微细钻头; 砂轮形状参数; 砂轮位置参数; 刃磨

中图分类号: TG713

\section{Research on Grinding Experiment of the Micro-drill Flute}

\section{LIANG Zhiqiang $^{1} \quad$ GUO Haixin $^{2} \quad$ ZHANG Suyan ${ }^{2} \quad$ WANG Xibin $^{1} \quad$ ZHOU Tianfeng $^{1}$ LIU Zhibing $^{1} \quad$ LI Yu $^{3}$ CHEN Jianjun ${ }^{3}$}

(1. Key Laboratory of Fundamental Science for Advanced Machining,

Beijing Institute of Technology, Beijing 100081;

2. School of Mechanical Engineering, Beijing Institute of Technology, Beijing 100081;

3. North General Power Group Co., Ltd., Datong 037036)

\begin{abstract}
Drill flute has a great influence on the drilling performance of micro-drill, and its profile largely depends on the grinding parameters, wheel profile and position. It is promising to obtain a desired flute profile with a standard wheel by adjusting the wheel position parameters. Based on the relative motion of drill flute and wheel, the grinding mathematical model of flute profile of micro-drill is proposed. Then considering the wheel profile and position, the flute profile is simulated by Matlab software. The radial rake angle and flute width are quantitatively calculated. The simulated results show that the wheel edge width, wheel inclined angle, wheel offset angle and distance have an obvious effect on the flute profile. With the increase of the wheel edge width and wheel inclined angle, the radial rake angle has no obvious change, but the flute width and chip space increase obviously. With the increased wheel offset angle, the radial rake angle increases obviously and the flute width decreases. However, with the increased wheel offset distance, the radial rake angle has no obvious change, but the flute width increases obviously. Based on the established mathematical model of grinding, micro drills with different flute profiles are fabricated, which confirms that the grinding results are in good agreement with the simulation results. The drilling experiment is carried out using the micro drills, which verified that the flute structure has an important influence on the chip removal capacity.
\end{abstract}

Key words: flute profile; micro-drill; wheel profile parameters; wheel position parameters; grinding

* 国家自然科学基金(51575049)、基础科研(DEDPHF)、国家重点基础研究 发展计划(973 计划, 2015CB059900)和微系统与微结构制造教育部重点 试验室开放课题研究(2015KM005)资助项目。20180824 收到初稿, 20190116 收到修改稿 


\section{0 前言}

螺旋槽是微细钻头的重要组成部分, 直接影 响刀具的切削性能和微孔的加工质量。螺旋槽结 构不仅决定了切削刃形状和刀具前角, 还影响着 微细钻头的容屑与排屑能力 ${ }^{[1]}$ 。螺旋槽形状与其刃 磨方法息息相关。在螺旋槽刃磨过程中, 通过砂 轮与刀具的相对螺旋运动包络形成螺旋槽曲面, 因此砂轮形状、砂轮位置和刃磨参数决定了螺旋 槽的几何结构。

近年来许多学者致力于研究螺旋槽的建模和加 工, 总的可以分成两种方法, 直接方法和间接方法。 其中间接方法是已知螺旋槽截形求解砂轮几何廓 形 ${ }^{[2-5]}$, 这种方法计算出的砂轮形状比较复杂, 难以 在实际中应用。直接方法是根据砂轮形状和位置参 数求解螺旋槽截面形状。将砂轮离散为一系列的薄 圆盘, 螺旋槽由若干个薄圆盘的切削轨迹叠加包络 形成 ${ }^{[6]}$ 。基于此种方法, 张伟等 ${ }^{[7]}$ 基于圆柱形砂轮, 提出了一种钻头螺旋槽的有效建模方法。BEJU 等 ${ }^{[8]}$ 建立了钻头螺旋槽的数学模型, 并分析了碟形砂轮 形状和砂轮位置参数对螺旋槽截形的影响。XIAO 等 ${ }^{[9]}$ 研究了砂轮形状和安装角度对螺旋槽径向前角 和槽宽的影响。

通过调整标准砂轮的位置参数可以获得所需的 螺旋槽形状, 因此, 许多学者开展了基于砂轮形状 和螺旋槽形状求解砂轮位置参数的研究。

KARPUSCHEWSKI 等 ${ }^{[10]}$ 开发了一种求解螺旋槽刃 磨过程中砂轮位置参数的自动搜索算法。 $\mathrm{TANG}$ 等 ${ }^{[11]}$ 为了刃磨出具有确定径向前角和钻芯厚度的螺旋槽 形状, 建立了求解砂轮位置参数的螺旋槽数学模型, 并提出了基于六轴数控工具磨床的雉面钻头刃磨方 法。REN 等 ${ }^{[12]}$ 提出了一种精确度较高的采用 $1 \mathrm{~V} 1 / 1 \mathrm{~A} 1$ 砂轮磨削端铣刀螺旋槽的加工方法, 这种 方法能有效地保证前角、钻芯厚度和螺旋槽宽度的 大小。张素燕等 ${ }^{[13]}$ 分析了砂轮与螺旋槽之间的相对 运动关系, 提出了微细钻头螺旋槽的数控加工方法, 实现了微细钻头螺旋槽的加工。何理论等 ${ }^{[14]}$ 基于无 瞬心包络原理提出了一种微细螺旋铣刀轴向型线的 计算模型, 计算过程与求解简单, 适用于对砂轮廓 形复杂时刃磨求解。

随着钻头直径的减小和高长径比的增加, 由螺 旋槽结构决定的容屑、排屑能力等越来越重要，因 此微细钻头螺旋槽结构不能简单地由传统钻头螺旋 槽结构缩放得到, 需要根据微细钻削的特点对其进 行设计分析。而开展微细钻头螺旋槽的建模与刃磨
研究是螺旋槽结构设计的前提条件。因此本研究首 先建立了微细钻头螺旋槽的数学模型, 并基于 Matlab 软件对螺旋槽横截面形状进行数值仿真。基 于仿真结果, 分析了砂轮形状与位置参数对螺旋槽 截形、径向前角以及槽宽的影响规律。为了验证数 学模型和仿真结果的正确性, 基于数控工具磨床进 行了微细钻头的刃磨试验研究。最后为了对比不同 螺旋槽型微细钻头的容屑与排屑能力, 开展微细钻 削试验研究。

\section{1 微细钻头螺旋槽型的刃磨数学模型}

\section{1 基于包络原理的螺旋槽几何建模}

钻头螺旋槽刃磨过程中, 砂轮与刀具的相对螺 旋运动形成螺旋槽曲面, 如图 1 所示。砂轮以速度 $\Omega_{\mathrm{w}}$ 绕其轴线的旋转运动作为切削主运动, 钻头以速 度 $\Omega_{\mathrm{d}}$ 和 $V_{\mathrm{d}}$ 绕其轴线旋转和平移运动作为螺旋运动。 $O_{\mathrm{w}} X_{\mathrm{w}} Y_{\mathrm{w}} Z_{\mathrm{w}}$ 为砂轮坐标系, 其中原点 $O_{\mathrm{w}}$ 位于砂轮大 端面的中心, $Z_{\mathrm{w}}$ 轴与砂轮轴线方向一致, $X_{\mathrm{w}}$ 轴和 $Y_{\mathrm{w}}$ 轴是位于砂轮大端面上相互垂直的两坐标轴。 $O_{\mathrm{f}} X_{\mathrm{f}} Y_{\mathrm{f}} Z_{\mathrm{f}}$ 是钻头坐标系, 其中 $X_{\mathrm{f}}$ 轴与 $X_{\mathrm{w}}$ 轴方向一致, $Z_{\mathrm{f}}$ 轴沿钻头轴线方向, $Z_{\mathrm{f}}$ 轴与 $Z_{\mathrm{w}}$ 轴之间的夹角为 $\lambda$ (砂轮偏置角度), 钻头中心与砂轮中心之间的偏移 距离为 $\left(a_{x}, a_{y}, a_{z}\right)$ 。坐标系 $O_{\mathrm{m}} X_{\mathrm{m}} Y_{\mathrm{m}} Z_{\mathrm{m}}$ 为刀具的动态 坐标系, 随着刀具的螺旋运动而动态变化。因此, 砂轮坐标系 $O_{\mathrm{w}} X_{\mathrm{w}} Y_{\mathrm{w}} Z_{\mathrm{w}}$ 通过坐标平移和绕 $X_{\mathrm{w}}$ 轴的 旋转可以得到钻头坐标系 $O_{\mathrm{f}} X_{\mathrm{f}} Y_{\mathrm{f}} Z_{\mathrm{f}}$, 坐标转换关系 式为

$$
\begin{gathered}
\boldsymbol{R}_{\mathrm{f}}=\operatorname{Rot}\left(X_{\mathrm{w}},-\lambda\right) \cdot \boldsymbol{R}_{\mathrm{w}}+\operatorname{Trans}\left(a_{x}, a_{y}, a_{z}\right)= \\
\left(\begin{array}{ccc}
1 & 0 & 0 \\
0 & \cos \lambda & -\sin \lambda \\
0 & \sin \lambda & \cos \lambda
\end{array}\right) \cdot \boldsymbol{R}_{\mathrm{w}}+\left(\begin{array}{l}
a_{x} \\
a_{y} \\
a_{z}
\end{array}\right)
\end{gathered}
$$

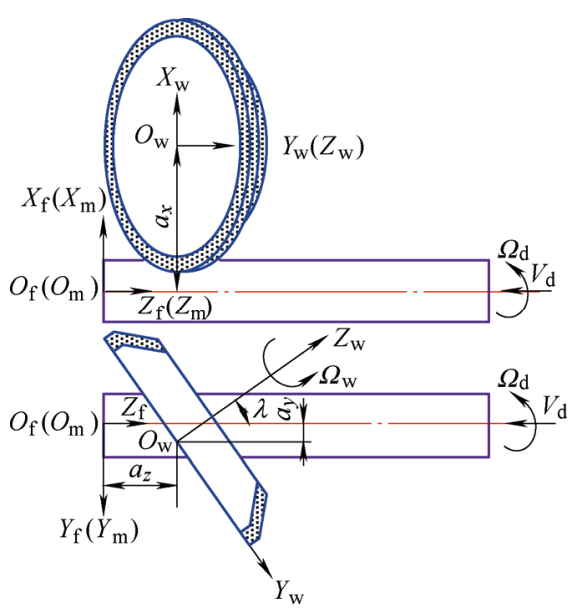

图 1 微细钻头螺旋槽刃磨过程示意图 
砂轮的几何结构如图 2 所示, 其中 $R_{0}$ 为砂轮半 径, $u_{1}$ 为砂轮宽度, $\eta$ 为砂轮雉角, $u_{2}$ 为砂轮边缘 宽度。在砂轮坐标系 $O_{\mathrm{w}} X_{\mathrm{w}} Y_{\mathrm{w}} Z_{\mathrm{w}}$ 中, 外轮廓面上任 意一点 $P$ 的坐标可以表示为

$$
\begin{aligned}
& \boldsymbol{R}_{\mathrm{w}}(u, \delta)=[R(u) \cos \delta, R(u) \sin \delta, u]^{\mathrm{T}} \\
& R(u)= \begin{cases}R_{0} & 0 \leqslant u \leqslant u_{2} \\
R_{0}-\left(u-u_{2}\right) \cot \eta & u_{2}<u \leqslant u_{1}\end{cases}
\end{aligned}
$$

式中, $u$ 和 $\delta$ 为点 $P$ 在砂轮外轮廓面上的位置参数。

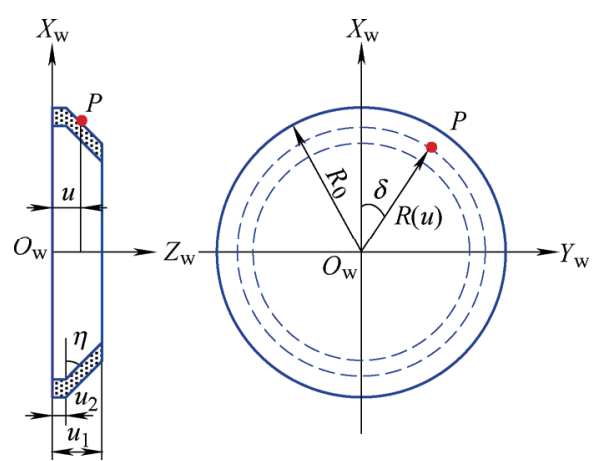

图 2 砂轮几何结构示意图

采用直接方法进行螺旋槽数学建模, 假设砂轮 由若干个薄截圆构成, 刃磨过程中每个薄截圆形成 不同的加工轨迹, 而螺旋槽曲面是由这些不同的加 工轨迹包络形成。假设点 $P$ 为任一薄截圆上的一点, 通过式(1)和(2)可以得到微细钻头坐标系 $O_{\mathrm{f}} X_{\mathrm{f}} Y_{\mathrm{f}} Z_{\mathrm{f}}$ 中 点 $P\left(X_{\mathrm{Pf}}, Y_{\mathrm{Pf}}, Z_{\mathrm{Pf}}\right)$ 的坐标为

$$
\boldsymbol{R}_{\mathrm{f}}(u, \delta)=\left[\begin{array}{c}
X_{\mathrm{Pf}} \\
Y_{\mathrm{Pf}} \\
Z_{\mathrm{Pf}}
\end{array}\right]=\left[\begin{array}{c}
R(u) \cos \delta+a_{x} \\
R(u) \sin \delta \cos \lambda-u \sin \lambda+a_{y} \\
R(u) \sin \delta \sin \lambda+u \cos \lambda+a_{z}
\end{array}\right] \text { (3) }
$$

根据螺旋槽刃磨加工原理, 砂轮相对于微细 钻头作螺旋运动, 假设砂轮绕 $O_{\mathrm{f}} Z_{\mathrm{f}}$ 轴旋转角度 $\varphi$, 则沿 $O_{\mathrm{f}} Z_{\mathrm{f}}$ 轴移动距离 $r \varphi / \tan \left(\beta_{0}\right)$, 其中 $r$ 是微细 钻头半径, $\beta_{0}$ 是螺旋槽的螺旋角。因此在钻头坐 标系 $O_{\mathrm{m}} X_{\mathrm{m}} Y_{\mathrm{m}} Z_{\mathrm{m}}$ 中, 点 $P\left(X_{\mathrm{Pm}}, Y_{\mathrm{Pm}}, Z_{\mathrm{Pm}}\right)$ 的加工轨 迹为

$$
\boldsymbol{R}_{\mathrm{m}}(u, \delta, \varphi)=\left[\begin{array}{c}
X_{\mathrm{Pm}} \\
Y_{\mathrm{Pm}} \\
Z_{\mathrm{Pm}}
\end{array}\right]=\left[\begin{array}{c}
X_{\mathrm{Pf}} \cos \varphi-Y_{\mathrm{Pf}} \sin \varphi \\
X_{\mathrm{Pf}} \sin \varphi+Y_{\mathrm{Pf}} \cos \varphi \\
Z_{\mathrm{Pf}}+r \varphi / \tan \left(\beta_{0}\right)
\end{array}\right]
$$

假设 $Z_{\mathrm{Pm}}=0$, 则 $\varphi=-Z_{\mathrm{Pf}} \tan \left(\beta_{0}\right) / r$, 点 $P$ 的加工轨 迹投影到平面 $Z_{\mathrm{Pm}}=0$ 上为

$$
\begin{gathered}
\boldsymbol{R}_{\mathrm{m}}(u, \delta)=\left[\begin{array}{c}
X_{\mathrm{pm}}(u, \delta) \\
Y_{\mathrm{pm}}(u, \delta)
\end{array}\right]=\left[\begin{array}{c}
X_{\mathrm{Pf}} \cos (\vartheta)-Y_{\mathrm{Pf}} \sin (\vartheta) \\
X_{\mathrm{Pf}} \sin (\vartheta)+Y_{\mathrm{Pf}} \cos (\vartheta)
\end{array}\right] \\
\vartheta=-Z_{\mathrm{Pf}} \tan \left(\beta_{0}\right) / r
\end{gathered}
$$

因此, 通过代入一系列的 $u$ 值和 $\delta$ 值, 可以得 到钻头横截面内若干个加工轨迹, 而螺旋槽横截面
形状则由这些加工轨迹包络形成。此外, 加工轨迹 上的点应该位于钻头横截面内, $X_{\mathrm{Pm}}$ 和 $Y_{\mathrm{Pm}}$ 坐标值应 该满足 $\left(X_{\mathrm{Pm}}{ }^{2}+Y_{\mathrm{Pm}}{ }^{2}\right) \leqslant r^{2}$ 。为了准确获得螺旋槽的横截 面形状, 根据文献[6]所列出的包络条件可以求解包 络曲线的数值解, 公式如下

$$
\frac{\partial X_{\mathrm{Pm}}}{\partial \delta} \times \frac{\partial Y_{\mathrm{Pm}}}{\partial u}-\frac{\partial Y_{\mathrm{Pm}}}{\partial \delta} \times \frac{\partial X_{\mathrm{Pm}}}{\partial u}=0
$$

将式(4)代入式(6), 可以获得包络曲线所应该满 足的关系式为

$$
\begin{gathered}
\frac{r}{\tan \beta_{0}}\left(\frac{\partial X_{\mathrm{Pf}}}{\partial \delta} \frac{\partial Y_{\mathrm{Pf}}}{\partial u}-\frac{\partial Y_{\mathrm{Pf}}}{\partial \delta} \frac{\partial X_{\mathrm{Pf}}}{\partial u}\right)+ \\
Y_{\mathrm{Pf}}\left(\frac{\partial Z_{\mathrm{Pf}}}{\partial \delta} \frac{\partial Y_{\mathrm{Pf}}}{\partial u}-\frac{\partial Y_{\mathrm{Pf}}}{\partial \delta} \frac{\partial Z_{\mathrm{Pf}}}{\partial u}\right)+ \\
X_{\mathrm{Pf}}\left(\frac{\partial Z_{\mathrm{Pf}}}{\partial \delta} \frac{\partial X_{\mathrm{Pf}}}{\partial u}-\frac{\partial X_{\mathrm{Pf}}}{\partial \delta} \frac{\partial Z_{\mathrm{Pf}}}{\partial u}\right)=0
\end{gathered}
$$

因此, 将 $u=0$ 代入式(5)中, 可以得到螺旋槽截 型非包络曲线的数值解，如图 3 所示。将不同的 $u$ 值代入式(7)中得到相应的 $\delta$ 角，进而将 $u$ 和 $\delta$ 值代 入式(5)可以得到包络曲线的数值解。

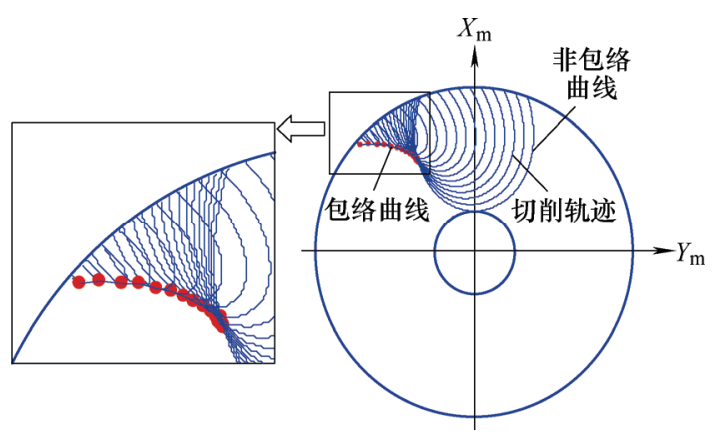

图 3 微细钻头螺旋槽截形

将坐标系 $O_{\mathrm{m}} X_{\mathrm{m}} Y_{\mathrm{m}} Z_{\mathrm{m}}$ 中螺旋槽的截型方程转换 到微细钻头坐标系 $O_{\mathrm{d}} X_{\mathrm{d}} Y_{\mathrm{d}} Z_{\mathrm{d}}$ 中, 如图 4 所示, 微细 钻头坐标系的原点 $O_{\mathrm{d}}$ 位于钻尖中心位置, $Z_{\mathrm{d}}$ 轴与微 细钻头轴线重合, $X_{\mathrm{d}}$ 轴方向满足使外缘转点 $C$ 的 $Y_{\mathrm{d}}$ 坐标为 $y_{\mathrm{c}}=t$ ( $2 t$ 为钻芯厚度)。因此微细钻头坐标 系中的螺旋槽截型的数值解可由下述坐标转换关系 式求得。

$$
\left\{\begin{array}{l}
{\left[\begin{array}{l}
x_{\mathrm{d}} \\
y_{\mathrm{d}} \\
z_{\mathrm{d}}
\end{array}\right]=\operatorname{Rot}\left(Z_{\mathrm{m}},-\frac{\pi}{2}+\chi\right) \operatorname{Rot}\left(X_{\mathrm{m}}, \pi\right)\left[\begin{array}{c}
x_{\mathrm{m}} \\
y_{\mathrm{m}} \\
z_{\mathrm{m}}
\end{array}\right]+\left[\begin{array}{l}
0 \\
0 \\
z_{\mathrm{c}}
\end{array}\right]} \\
\chi=\arctan \left(\frac{x_{\mathrm{cm}}}{y_{\mathrm{cm}}}\right)-\arcsin \left(\frac{t}{r}\right)
\end{array}\right.
$$

式中, $\left(x_{\mathrm{cm}}, y_{\mathrm{cm}}\right)$ 是外缘转点 $C$ 在坐标系 $O_{\mathrm{m}} X_{\mathrm{m}} Y_{\mathrm{m}} Z_{\mathrm{m}}$ 中的坐标值, $z_{\mathrm{c}}$ 是 $C$ 点在坐标系 $O_{\mathrm{d}} X_{\mathrm{d}} Y_{\mathrm{d}} Z_{\mathrm{d}}$ 中的坐 标值。 


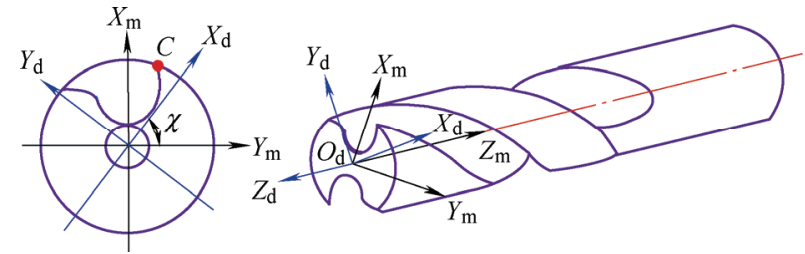

图 4 坐标转换示意图

对上述所求得的螺旋槽截型数值解进行三次样 条插值处理, 得到螺旋槽截型方程为: $y_{\mathrm{d}}=f_{\mathrm{H}}\left(x_{\mathrm{d}}\right)$ 。螺 旋槽曲面是由螺旋槽截型作螺旋运动形成, 因此可 以得到螺旋槽数学方程为

$$
F_{0}:\left\{\begin{array}{l}
X_{\mathrm{d}}=x_{\mathrm{d}} \cos y_{\mathrm{d}}-f_{\mathrm{H}}\left(x_{\mathrm{d}}\right) \sin y_{\mathrm{d}} \\
Y_{\mathrm{d}}=x_{\mathrm{d}} \sin y_{\mathrm{d}}+f_{\mathrm{H}}\left(x_{\mathrm{d}}\right) \cos y_{\mathrm{d}} \\
Z_{\mathrm{d}}=z_{\mathrm{c}}+r y_{\mathrm{d}} / \tan \beta_{0}
\end{array}\right.
$$

\section{2 微细钻头螺旋槽几何角度建模}

为了评价砂轮形状、位置参数对螺旋槽截形的 影响, 对螺旋槽径向前角和螺旋槽宽度进行数值求 解 ${ }^{[15]}$ 。径向前角 $\gamma$ 是矢量 $\boldsymbol{O}_{\mathrm{m}} \boldsymbol{P}_{1}$ 与切矢量 $\boldsymbol{P}_{1} \boldsymbol{P}_{3}$ 之间 的夹角, 螺旋槽宽度 $\alpha$ 为矢量 $\boldsymbol{O}_{\mathrm{m}} \boldsymbol{P}_{1}$ 与矢量 $\boldsymbol{O}_{\mathrm{m}} \boldsymbol{P}_{2}$ 之 间的夹角, 如图 5 所示。点 $P_{1}$ 和 $P_{2}$ 位于外圆上, 因此坐标满足关系式

$$
\left\{\begin{array}{l}
X_{\mathrm{m}}^{2}\left(u_{1}, \delta_{1}\right)+Y_{\mathrm{m}}^{2}\left(u_{1}, \delta_{1}\right)=r^{2} \\
X_{\mathrm{m}}^{2}\left(u_{2}, \delta_{2}\right)+Y_{\mathrm{m}}^{2}\left(u_{2}, \delta_{2}\right)=r^{2}
\end{array}\right.
$$

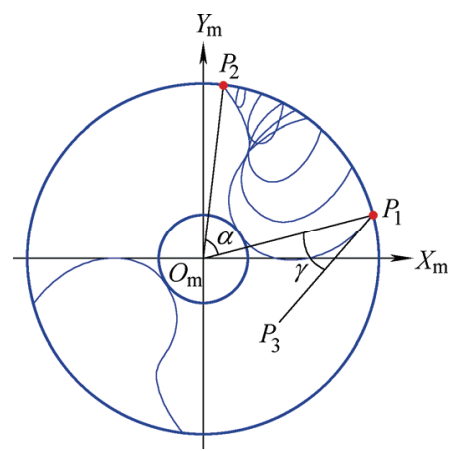

图 5 径向前角 $\gamma$ 和螺旋槽宽度 $\alpha$ 示意图

此外, 点 $P_{1}$ 位于非包络曲线上, 将 $u_{1}=0$ 代入 式(5)和(10)可以求得点 $P_{1}$ 的坐标值。点 $P_{2}$ 位于包络 曲线上, 联立式(5)、(7)和(10)可以求得点 $P_{2}$ 的坐标 值。因此矢量 $\boldsymbol{O}_{\mathrm{m}} \boldsymbol{P}_{1}$ 和 $\boldsymbol{O}_{\mathrm{m}} \boldsymbol{P}_{2}$ 可以表示为

$$
\left\{\begin{array}{l}
\boldsymbol{O}_{\mathrm{m}} \boldsymbol{P}_{1}=\left[X_{\mathrm{m}}\left(u_{1}, \delta_{1}\right), Y_{\mathrm{m}}^{2}\left(u_{1}, \delta_{1}\right), 0\right]^{\mathrm{T}} \\
\boldsymbol{O}_{\mathrm{m}} \boldsymbol{P}_{2}=\left[X_{\mathrm{m}}\left(u_{2}, \delta_{2}\right), Y_{\mathrm{m}}^{2}\left(u_{2}, \delta_{2}\right), 0\right]^{\mathrm{T}}
\end{array}\right.
$$

矢量 $\boldsymbol{P}_{1} \boldsymbol{P}_{3}$ 为非包络曲线段的切矢量, 可以表 示为

$$
\boldsymbol{P}_{1} \boldsymbol{P}_{3}=\left[\frac{\partial X_{\mathrm{m}}\left(u_{1}, \delta\right)}{\partial \delta}, \frac{\partial Y_{\mathrm{m}}\left(u_{1}, \delta\right)}{\partial \delta}, 0\right]_{\delta=\delta_{1}}^{\mathrm{T}}
$$

因此, 径向前角 $\gamma$ 和螺旋槽宽度 $\alpha$ 可以通过下 述方程求得

$$
\gamma=\arccos \frac{\boldsymbol{O}_{\mathrm{m}} \boldsymbol{P}_{1} \cdot \boldsymbol{P}_{1} \boldsymbol{P}_{3}}{\left|\boldsymbol{O}_{\mathrm{m}} \boldsymbol{P}_{1}\right|\left|\boldsymbol{P}_{1} \boldsymbol{P}_{3}\right|}, \alpha=\arccos \frac{\boldsymbol{O}_{\mathrm{m}} \boldsymbol{P}_{1} \cdot \boldsymbol{O}_{\mathrm{m}} \boldsymbol{P}_{2}}{\left|\boldsymbol{O}_{\mathrm{m}} \boldsymbol{P}_{1}\right|\left|\boldsymbol{O}_{\mathrm{m}} \boldsymbol{P}_{2}\right|}
$$

\section{2 螺旋槽截形 Matlab 数值仿真}

基于 Matlab 仿真软件, 对螺旋槽截形进行数值 求解。微细钻头几何结构参数、砂轮形状参数和位 置参数作为输入量代入仿真程序中, 它们分别为钻 头直径 $d$ 、钻芯厚度 $2 t$ 、螺旋角 $\beta_{0}$ 、砂轮半径 $R_{0}$ 、 砂轮宽度 $u_{1}$ 、砂轮锥角 $\eta$ 、砂轮边缘宽度 $u_{2}$ 、砂轮 偏置角度 $\lambda$ 以及砂轮中心与钻头中心之间的偏移距 离 $a_{x} 、 a_{y} 、 a_{z}$, 如表 1 所示。

表 1 微细钻头几何参数和砂轮形状与位置参数

\begin{tabular}{lc||cc}
\hline \multicolumn{1}{c|}{ 参数 } & 数值 & 参数 & 数值 \\
\hline 钻头直径 $d / \mathrm{mm}$ & 0.5 & 砂轮边缘宽度 $u_{2} / \mathrm{mm}$ & $0 \sim 0.15$ \\
钻芯厚度 $2 t / \mathrm{mm}$ & 0.125 & 砂轮偏移距离 $a_{x} / \mathrm{mm}$ & 67.5625 \\
螺旋角 $\beta_{0} /\left(^{\circ}\right)$ & 30 & 砂轮偏移距离 $a_{y} / \mathrm{mm}$ & $0 \sim 9$ \\
砂轮半径 $R_{0} / \mathrm{mm}$ & 67.5 & 砂轮偏移距离 $a_{z} / \mathrm{mm}$ & 0 \\
砂轮宽度 $u_{1} / \mathrm{mm}$ & 4 & 砂轮偏置角度 $\lambda /\left(^{\circ}\right)$ & $30 \sim 60$ \\
砂轮锥角 $\eta /\left(^{\circ}\right)$ & $35 \sim 65$ & & \\
\hline
\end{tabular}

砂轮形状参数包括砂轮半径 $R_{0}$ 、砂轮宽度 $u_{1}$ 、 砂轮锥角 $\eta$ 、砂轮边缘宽度 $u_{2}$, 本研究主要分析讨 论砂轮锥角 $\eta$ 和砂轮边缘宽度 $u_{2}$ 对螺旋槽截形的影 响规律。不同砂轮形状参数下, 螺旋槽截形仿真结 果如图 6、7 所示, 砂轮形状参数对径向前角和宽度

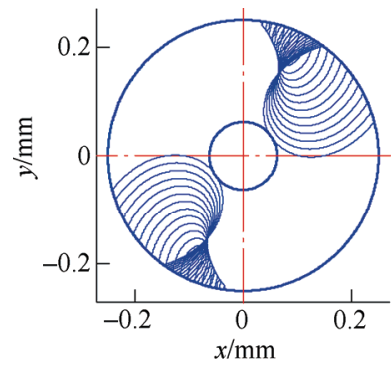

(a) $u_{2}=0 \mathrm{~mm}$

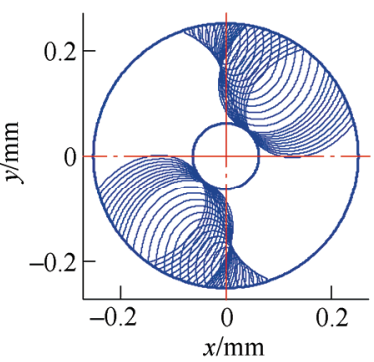

(c) $u_{2}=0.1 \mathrm{~mm}$

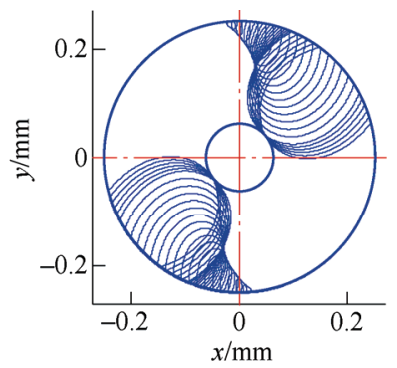

(b) $u_{2}=0.05 \mathrm{~mm}$

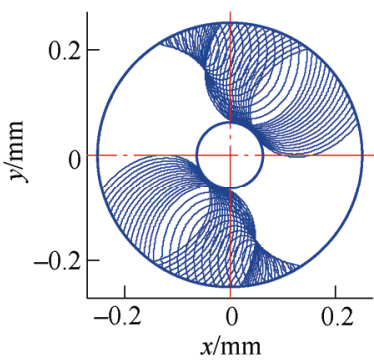

(d) $u_{2}=0.15 \mathrm{~mm}$
图 6 砂轮边缘宽度 $u_{2}$ 对螺旋槽截形的影响 $\left(\eta=45^{\circ}, \lambda=60^{\circ}, a_{x}=67.5625 \mathrm{~mm}, a_{y}=0\right)$ 


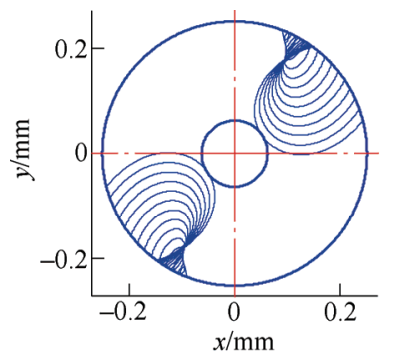

(a) $\eta=35^{\circ}$

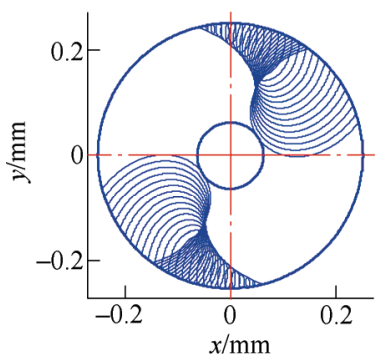

(c) $\eta=55^{\circ}$

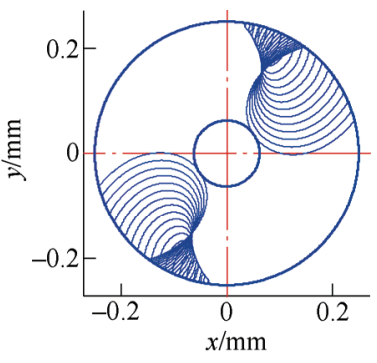

(b) $\eta=45^{\circ}$

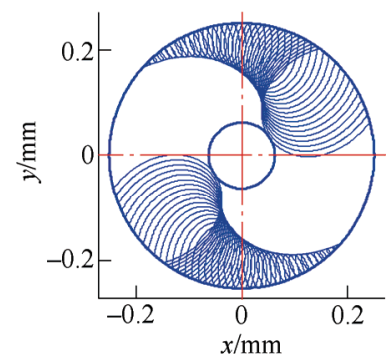

(d) $\eta=65^{\circ}$
图 7 砂轮雉角 $\eta$ 对螺旋槽截形的影响

$\left(u_{2}=0, \lambda=60^{\circ}, a_{x}=67.5625 \mathrm{~mm}, a_{y}=0\right)$

的影响规律如图 8 所示。可以看出砂轮锥角 $\eta$ 、砂 轮边缘宽度 $u_{2}$ 对螺旋槽截形有明显的影响。随着砂 轮边缘宽度 $u_{2}$ 、砂轮堆角 $\eta$ 的增大, 径向前角没有 变化, 但是螺旋槽宽度增大, 容屑空间明显增大。

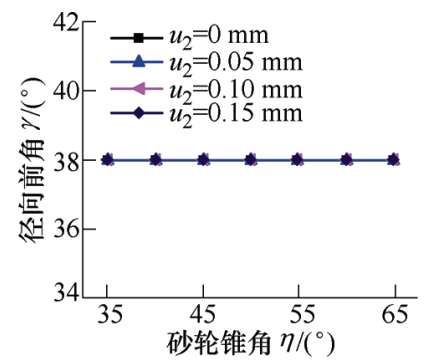

(a) 径向前角 $\gamma$

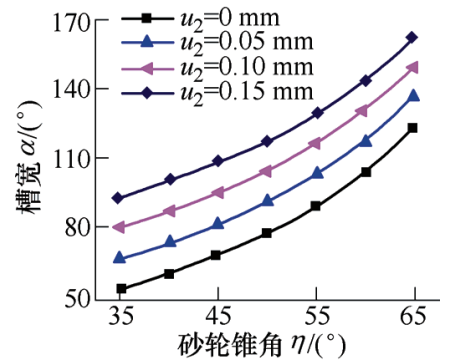

(b) 螺旋槽宽度 $\alpha$

图 8 砂轮形状参数对螺旋槽径向前角和螺旋槽宽度的影响

砂轮位置参数包括砂轮偏置角度 $\lambda$ 和砂轮偏移 距离 $a_{x} 、 a_{y} 、 a_{z}$, 其中偏移距离 $a_{x} 、 a_{z}$ 对于等芯厚 等槽型的螺旋槽截形没有影响, 因此主要分析讨论 砂轮偏置角度 $\lambda$ 和偏移距离 $a_{y}$ 对螺旋槽截形的影响 规律。不同砂轮位置参数下, 螺旋槽截形仿真结果 如图 9、10 所示。可以看出位置参数 $\lambda 、 a_{y}$ 对螺旋 槽截形有显著的影响。随着砂轮偏置角度 $\lambda$ 的减小,
螺旋槽容屑空间增大, 螺旋槽截形为传统的槽型。 随着砂轮偏移距离 $a_{y}$ 的增大, 螺旋槽容屑空间也增 大, 螺旋槽截形变为抛物线型截形。

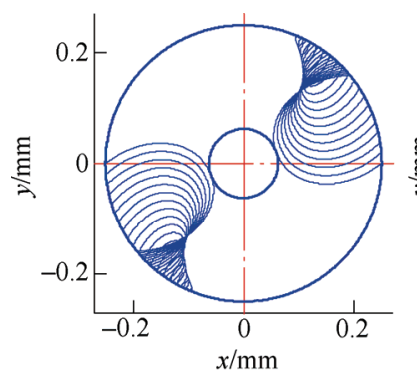

(a) $\lambda=60^{\circ}$

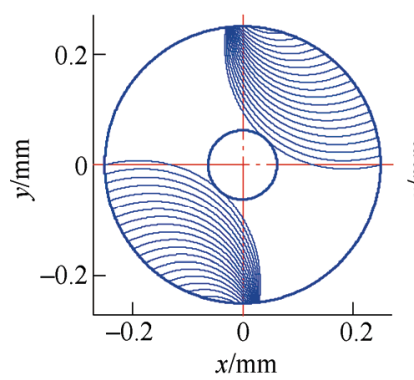

(c) $\lambda=40^{\circ}$

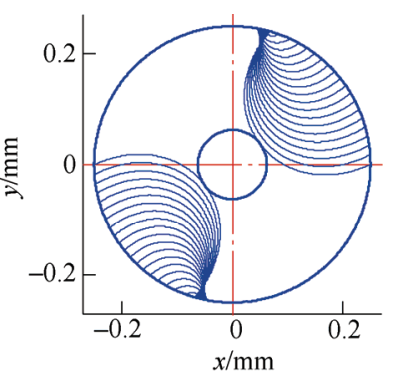

(b) $\lambda=50^{\circ}$

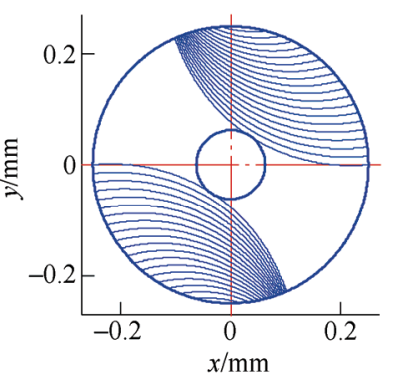

(d) $\lambda=30^{\circ}$
图 9 砂轮安装角 $\lambda$ 对螺旋槽截形的影响 $\left(u_{2}=0, \eta=45^{\circ}, a_{x}=67.5625 \mathrm{~mm}, a_{y}=0\right)$

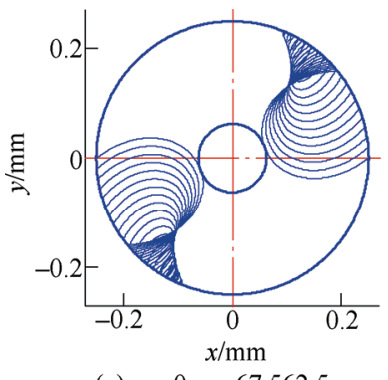

(a) $a_{y}=0, a_{x}=67.5625 \mathrm{~mm}$

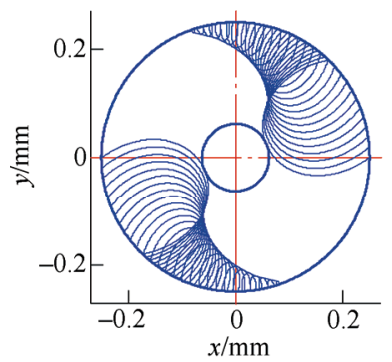

(c) $a_{y}=6 \mathrm{~mm}, a_{x}=66.4912 \mathrm{~mm}$

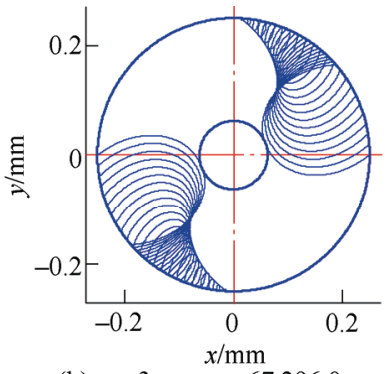

(b) $a_{y}=3 \mathrm{~mm}, a_{x}=67.2960 \mathrm{~mm}$

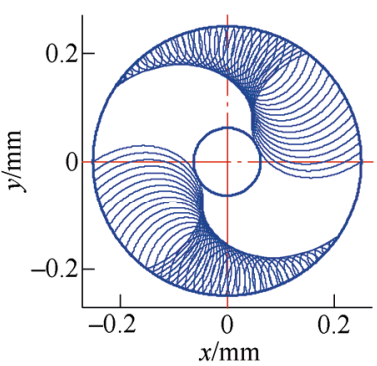

(d) $a_{y}=9 \mathrm{~mm}, a_{x}=65.1272 \mathrm{~mm}$
图 10 砂轮偏移距离 $a_{y}$ 对螺旋槽截形的影响

$$
\left(u_{2}=0, \eta=45^{\circ}, \lambda=60^{\circ}\right)
$$

砂轮位置参数对径向前角和螺旋槽宽度的影响 如图 11 所示, 可以看出随着砂轮偏置角 $\lambda$ 的增大, 径向前角 $\gamma$ 有明显的增大趋势。而随着砂轮偏移距 离 $a_{y}$ 的增大，径向前角 $\gamma$ 有轻微的减小趋势。对于 较大的砂轮安装角 $\lambda\left(\lambda=60^{\circ} 、 50^{\circ}\right)$, 砂轮偏移距离 $a_{y}$ 的增大引起螺旋槽宽度 $\alpha$ 的明显增大。而对于较小 的砂轮安装角度 $\lambda\left(\lambda=40^{\circ} 、 30^{\circ}\right)$, 随着参数 $a_{y}$ 的增大, 
螺旋槽宽度 $\alpha$ 没有明显的变化。此外, 当 $a_{y}=0$ 时, 螺旋槽宽度 $\alpha$ 与砂轮安装角 $\lambda$ 成反比关系, 而当 $a_{y}$ 不等于 0 时, 螺旋槽宽度 $\alpha$ 与 $\lambda$ 没有明显的比例关 系。因此, 砂轮偏置角度对螺旋槽形状及其径向前 角和螺旋槽宽度有明显的影响, 而砂轮偏移距离对 螺旋槽形状和螺旋槽宽度有明显的影响。

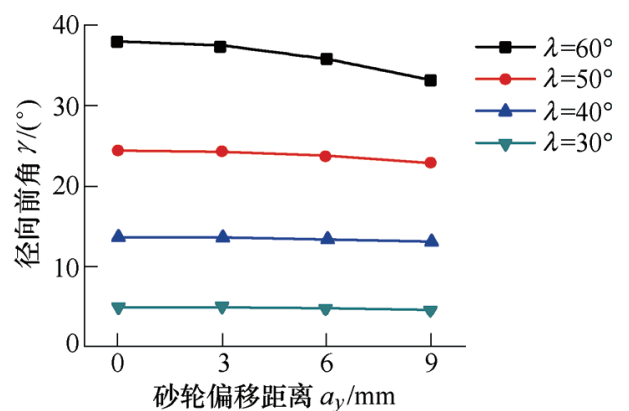

(a) 径向前角 $\gamma$

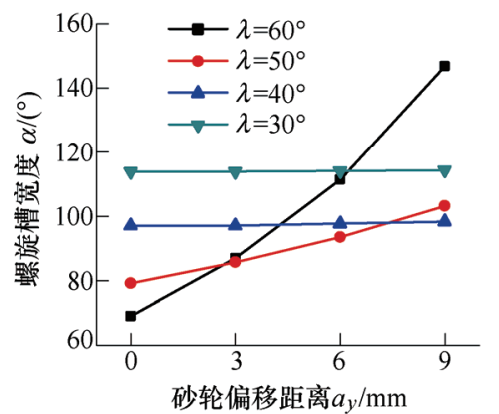

(b) 螺旋槽宽度 $\alpha$

图 11 不同砂轮位置参数下螺旋槽径向前角和螺旋槽宽度

\section{3 微细钻头刃磨试验}

\section{1 微细钻头刃磨制备试验条件}

为了验证上述建模方法和仿真结果的正确性及 有效性, 开展不同砂轮位置参数的微细钻头刀磨试 验研究。采用牧野精机 CNS7d 六轴数控工具磨床对 微细钻头螺旋槽进行刃磨制备, 该机床定位精度为 $0.0015 \mathrm{~mm}$, 重复定位精度为 $0.002 \mathrm{~mm}$, 运动原理 如图 12 所示。在刃磨过程中, 微细钻头装卡在 $A$ 轴上, 能实现沿 $Y$ 轴、 $U$ 轴的移动以及绕 $W$ 轴、 $A$ 轴的旋转, 与此同时砂轮能实现沿 $X$ 轴和 $Z$ 轴移动。

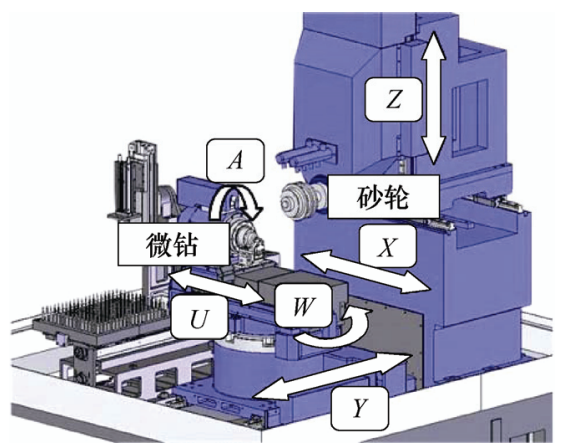

图 12 数控工具磨床各轴运动示意图
微细钻头刃磨试验装置如图 13 所示。砂轮选用 日本住友金刚石砂轮, 砂轮结合剂为陶瓷, 砂轮粒 度为 2000。采用单斜边砂轮加工微细钻头螺旋槽, 形状参数为直径 $D_{\mathrm{w} 1}=135 \mathrm{~mm}$, 砂轮宽度 $u_{1}=4 \mathrm{~mm}$, 砂轮雉角 $\eta=\pi / 4$, 边缘宽度 $u_{2}=0$ 。采用平行砂轮完 成微细钻头后刀面的加工。微细钻头磨制过程中砂 轮的磨削速度为 $24 \mathrm{~m} / \mathrm{s}$, 粗加工时的磨削深度为 20 $\mu \mathrm{m}$, 精加工时的磨削深度为 $4 \mu \mathrm{m}$, 螺旋槽加工时 的进给速度为 $13 \mathrm{~mm} / \mathrm{s}$, 磨削过程采用磨削油冷却 磨削。微细钻头材料采用超细颗粒度硬质合金 DK500UF, WC 晶粒度为 $0.5 \mu \mathrm{m}$, WC 晶粒的细化 增强了硬质合金材料的硬度和强度, 材料化学成分 和物理特性如表 2 所示。

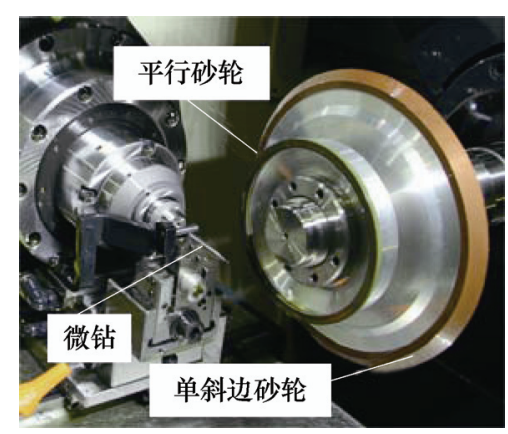

图 13 微细钻头刃磨试验装置

表 2 硬质合金微细钻头材料化学成分和物理特性

\begin{tabular}{ccccc}
\hline 硬度 & $\begin{array}{c}\text { 密度 } \\
\mathrm{HV} /\left(\mathrm{N} / \mathrm{mm}^{2}\right)\end{array}$ & $\begin{array}{c}\text { 晶粒尺寸 } \\
/\left(\mathrm{g} / \mathrm{cm}^{3}\right)\end{array}$ & \multicolumn{2}{c}{ 重量百分比 $(\%)$} \\
\cline { 4 - 6 } & $14 \mathrm{~m}$ & $\mathrm{WC}$ & $\mathrm{Co}$ \\
\hline 1660 & 14.15 & 0.5 & 88 & 12 \\
\hline
\end{tabular}

\section{2 螺旋槽刃磨初始位置控制}

微细钻头几何形状是由螺旋槽磨削加工和后刀 面磨削加工两个工序共同决定, 而主切削刃曲线是 由螺旋槽曲面和后刀面相交形成。因此为了控制主 切削刃曲线形状, 在实现螺旋槽和后刀面几何形状 精确磨削的基础上，需要控制螺旋槽磨削后主切削 刃外缘转点在微细钻头外圆柱面上的位置, 这样经 过后续的后刀面磨削能够形成特定的切削刃曲线。

当砂轮的几何参数确定时, 螺旋槽曲面由砂轮 的位置参数决定。砂轮位置参数包括砂轮偏置角度 $\lambda$ 和砂轮偏移距离 $a_{x} 、 a_{y} 、 a_{z}$, 其中砂轮偏移距离 $a_{z}$ 对螺旋槽横截面形状没有影响, 但是决定了主切削 刃外缘转点 $C$ 在微细钻头外圆柱面上的位置, 如图 14 所示。图 15 为不同砂轮偏移距离 $a_{z}$ 所获得的 $Z_{\mathrm{m}}=0.15 \mathrm{~mm}$ 平面上的螺旋槽横截面形状, 随着参数 $a_{z}$ 的改变, $C$ 点的坐标发生了变化。由微细钻头几 何结构尺寸可知, 主切削刃外缘转点 $C$ 点的坐标为 $C\left(x_{\mathrm{c}}, y_{\mathrm{c}}, z_{\mathrm{c}}\right)=\left(\sqrt{r^{2}-t^{2}}, t, z_{\mathrm{c}}\right)$, 由于主切削刃外缘转 
点 $C$ 位于后刀面上, 因此 $z_{\mathrm{c}}$ 值可由后刀面确定。

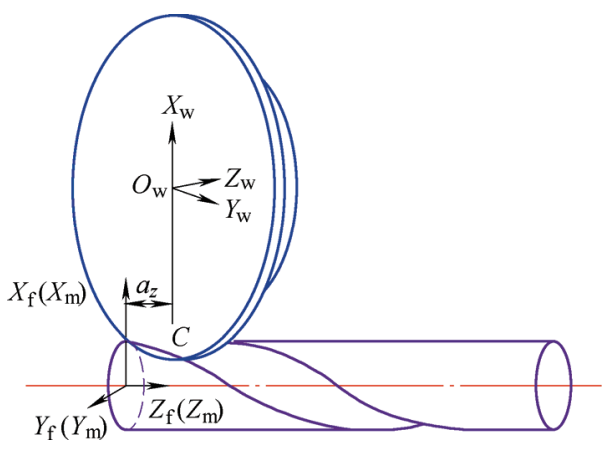

图 14 主切削刃外缘转点 $C$ 的位置控制

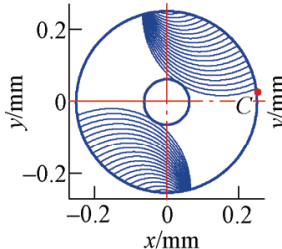

(a) $a_{z}=-0.1 \mathrm{~mm}$

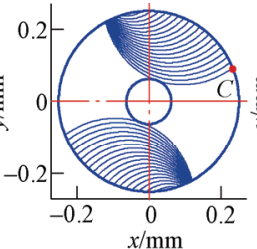

(b) $a_{z}=0 \mathrm{~mm}$

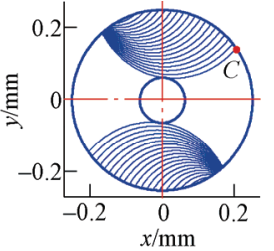

(c) $a_{z}=0.1 \mathrm{~mm}$
图 15 砂轮偏移距离 $a_{z}$ 对主切削刃外缘转点 $C$ 位置的影响

主切削刃外缘转点 $C$ 处的螺旋槽曲面是由砂轮 大端面磨削形成, 而砂轮大端面的轮廓方程为

$R_{\mathrm{w}}(\delta)=\left[X_{\mathrm{Pw}_{\mathrm{w}}}, Y_{\mathrm{Pw}_{\mathrm{w}}}, Z_{\mathrm{Pw}_{\mathrm{w}}}\right]^{\mathrm{T}}=[R \cos \delta, R \sin \delta, 0]^{\mathrm{T}}$

经过砂轮与钻头之间的坐标转换以及钻头绕自 身轴线的螺旋运动转换, 得到砂轮大端面在坐标系 $O_{\mathrm{m}} X_{\mathrm{m}} Y_{\mathrm{m}} Z_{\mathrm{m}}$ 中的坐标为

$$
\begin{gathered}
R_{\mathrm{m}}\left(\delta, \varphi, a_{z}\right)=\left[\begin{array}{c}
X_{\mathrm{Pm}} \\
Y_{\mathrm{Pm}} \\
Z_{\mathrm{Pm}}
\end{array}\right]=\left[\begin{array}{c}
X_{\mathrm{Pf}} \cos \varphi-Y_{\mathrm{Pf}} \sin \varphi \\
X_{\mathrm{Pf}} \sin \varphi+Y_{\mathrm{Pf}} \cos \varphi \\
Z_{\mathrm{Pf}}+d \varphi / 2 \tan \left(\beta_{0}\right)
\end{array}\right] \\
R_{\mathrm{f}}\left(\delta, a_{z}\right)=\left[\begin{array}{c}
X_{\mathrm{Pf}} \\
Y_{\mathrm{Pf}} \\
Z_{\mathrm{Pf}}
\end{array}\right]=\left[\begin{array}{c}
R \cos \delta+a_{x} \\
R \sin \delta \cos \lambda+a_{y} \\
R \sin \delta \sin \lambda+a_{z}
\end{array}\right]
\end{gathered}
$$

当 $Z_{\mathrm{Pm}}=z_{\mathrm{c}}$ 时, $\varphi=\left(z_{\mathrm{c}}-Z_{\mathrm{Pf}}\right) \tan \left(\beta_{0}\right) / r$, 则砂轮 大端面轮廓在平面 $Z_{\mathrm{Pm}}=z_{\mathrm{c}}$ 上的坐标为

$R_{\mathrm{m}}\left(\delta, a_{z}\right)=\left[\begin{array}{c}X_{\mathrm{Pm}}\left(\delta, a_{z}\right) \\ Y_{\mathrm{Pm}}\left(\delta, a_{z}\right)\end{array}\right]=\left[\begin{array}{l}X_{\mathrm{Pf}} \cos v-Y_{\mathrm{Pf}} \sin v \\ X_{\mathrm{Pf}} \sin v+Y_{\mathrm{Pf}} \cos v\end{array}\right]$

式中, $v=\left(z_{\mathrm{c}}-Z_{\mathrm{Pf}}\right) \tan \left(\beta_{0}\right) / r$ 。

因此通过联立方程式(16)即可求得砂轮偏移距 离 $a_{z}$ 的值。

$$
X_{\mathrm{Pm}}\left(\delta, a_{z}\right)=\sqrt{r^{2}-t^{2}} \quad Y_{\mathrm{Pm}}\left(\delta, a_{z}\right)=t
$$

此时求得的 $a_{z}$ 值为以螺旋槽导程的一半为间距 的一系列数据点, 当 $a_{z}>0$ 时, 螺旋槽磨削完成后存 在一未加工区域, 如图 16 所示, 将会影响后刀面以 及主切削刃的形状, 因此在求解式(16)时需保证 $a_{z}<0$ 。
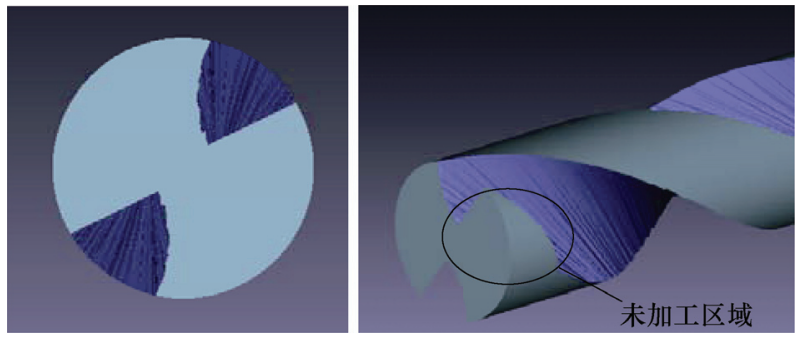

图 16 螺旋槽磨削加工初始位置控制

\section{3 微细钻头刃磨试验研究}

螺旋槽刃磨过程如图 17 所示, $L_{X} 、 L_{Y} 、 L_{Z}$ 为钻 尖中心 $O_{\mathrm{d}}$ 到机床原点 $O$ 沿 $X 、 Y 、 Z$ 轴的距离。磨 削开始之前, $X 、 Y 、 Z$ 和 $W$ 轴移动旋转至螺旋槽磨 削初始位置, 即 $W$ 轴旋转角度 $90^{\circ}-\lambda$, 微细钻头沿 $Y$ 轴移动距离 $y_{1}=L_{Y}+a_{z} \cos \lambda-a_{y} \sin \lambda$, 砂轮沿 $X 、 Z$ 轴 移动距离分别为 $x_{1}=L_{X}+a_{z} \sin \lambda+a_{y} \cos \lambda, z_{1}=L_{Z}-a_{x}$, 其 中 $\lambda 、 a_{x} 、 a_{y} 、 a_{z}$ 为砂轮位置参数。刀磨制备 $d=0.5$ $\mathrm{mm} 、 2 t=0.125 \mathrm{~mm} 、 \beta_{0}=30^{\circ}$ 的微细钻头螺旋槽, 相 应的砂轮位置参数分别为 $\lambda=40^{\circ} 、 50^{\circ} 、 60^{\circ}, a_{x}=$ $67.5625 \mathrm{~mm}, a_{y}=0, a_{z}$ 值由式(15)求解得到。磨 削时 $U$ 轴和 $A$ 轴分别进行直线与旋转的插补运 动, 即 $A$ 轴旋转角度 $\varphi$ 的同时 $U$ 轴移动距离 $d \varphi / 2 \tan \left(\beta_{0}\right)$, 其中 $d$ 为微细钻头的直径, $\beta_{0}$ 为螺旋 槽的螺旋角。通过 $U$ 轴和 $A$ 轴的联合运动形成一 个完整螺旋槽。

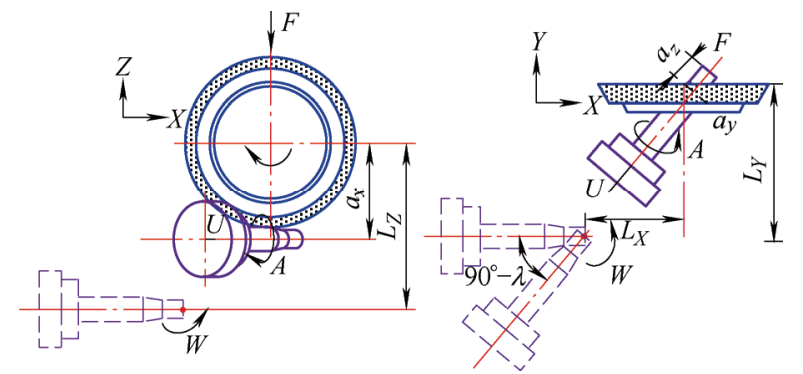

图 17 微细钻头螺旋槽刃磨过程示意图

基于牧野 CNS7d 数控磨床磨制直径 $0.5 \mathrm{~mm}$ 的 硬质合金微细钻头螺旋槽，不同砂轮偏置角度所对 应的螺旋槽刃磨试验结果如图 18 所示。刀磨试验结 果与 Mtalab 仿真结果进行对比, 图 19 为不同砂轮 偏置角度所形成的螺旋槽型仿真结果, 发现试验结 果与仿真结果具有较好的一致性。为了保证试验的 准确性和可重复性, 针对于不同的砂轮位置参数开 展 3 次刃磨试验, 使用基恩士 VK-X100 对螺旋槽几 何参数进行测量。 3 把刀具几何参数的实测平均值 和 Matlab 仿真值如表 3 所示, 其中微细钻头基本几 何结构参数 $(d 、 2 t)$ 的误差小于 $2 \%$, 径向前角 $\gamma$ 和槽 宽 $\alpha$ 的误差在 5\% 17\%之间, 进一步验证了基于包 络原理采用解析方法所建立的螺旋槽横截面数学模 
型和仿真结果的正确性。其中几何结构参数的试验 误差是由于在实际磨削过程中, 砂轮产生微量磨损 使砂轮的边缘宽度 $u_{2}$ 未能精确保持为 0 。最后不同 槽型微细钻头刃磨结果如图 20 所示。

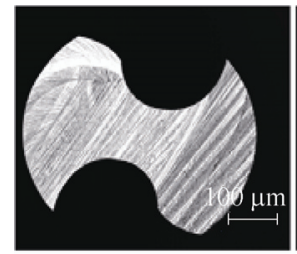

(a) $\lambda=60^{\circ}$

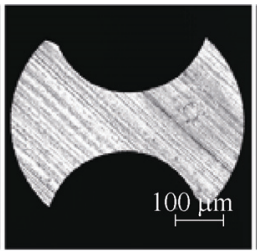

(b) $\lambda=50^{\circ}$

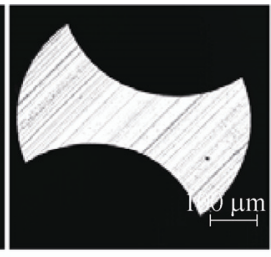

(c) $\lambda=40^{\circ}$
图 18 螺旋槽刃磨试验结果

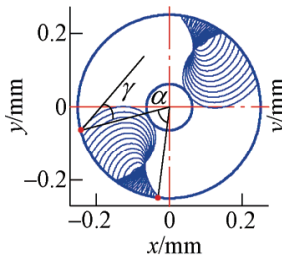

(a) $\lambda=60^{\circ}$

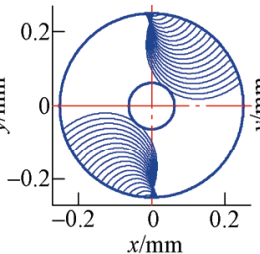

(b) $\lambda=50^{\circ}$

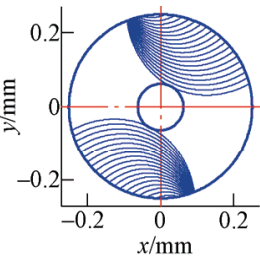

(c) $\lambda=40^{\circ}$
图 19 螺旋槽图解法 Matlab 仿真结果

表 3 螺旋槽径向前角 $\gamma$ 和槽宽 $\alpha$ 的仿真与刀磨结果对比

\begin{tabular}{|c|c|c|c|c|c|}
\hline $\begin{array}{c}\text { 砂轮偏置 } \\
\text { 角度 } \lambda\end{array}$ & & $\begin{array}{c}\text { 钻头直径 } \\
d / \mu \mathrm{m}\end{array}$ & $\begin{array}{c}\text { 钻芯厚度 } \\
2 t / \mu \mathrm{m}\end{array}$ & $\begin{array}{c}\text { 径向前角 } \\
\gamma /\left(^{\circ}\right)\end{array}$ & $\begin{array}{l}\text { 槽宽 } \\
\left.\alpha / /^{\circ}\right) \\
\end{array}$ \\
\hline \multirow{3}{*}{$\lambda=60^{\circ}$} & 仿真值 & 500 & 125 & 37.96 & 68.96 \\
\hline & $\begin{array}{c}\text { 实测 } \\
\text { 平均值 }\end{array}$ & 502.82 & 125.67 & 34.76 & 80.01 \\
\hline & 误差(\%) & 0.56 & 0.54 & 8.46 & 16.02 \\
\hline \multirow{3}{*}{$\lambda=50^{\circ}$} & 仿真值 & 500 & 125 & 24.52 & 79.22 \\
\hline & $\begin{array}{c}\text { 实测 } \\
\text { 平均值 }\end{array}$ & 501.53 & 126.90 & 25.90 & 87.30 \\
\hline & 误差(\%) & 0.31 & 1.52 & 5.63 & 10.20 \\
\hline \multirow{3}{*}{$\lambda=40^{\circ}$} & 仿真值 & 500 & 125 & 13.77 & 96.99 \\
\hline & $\begin{array}{c}\text { 实测 } \\
\text { 平均值 }\end{array}$ & 503.05 & 126.81 & 12.11 & 102.37 \\
\hline & 误差(\%) & 0.61 & 1.45 & 12.06 & 5.55 \\
\hline
\end{tabular}

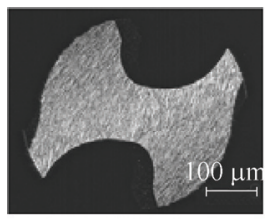

(a) 刀具 $1\left(\lambda=60^{\circ}\right)$

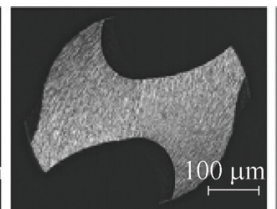

(b) 刀具 $2\left(\lambda=50^{\circ}\right)$

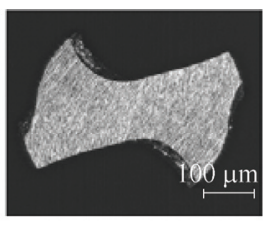

(c) 刀具 $3\left(\lambda=40^{\circ}\right)$
图 20 微细钻头刃磨试验结果

\section{4 微细钻头的钻削试验研究}

基于德国德玛吉机床有限公司生产的 DMU80 monoBLOCK 加工中心, 采用所制备的微细钻头, 对 $1 \mathrm{Cr} 18 \mathrm{Ni} 9 \mathrm{Ti}$ 进行微小孔钻削试验研究。微小孔试 验装置如图 21 所示。

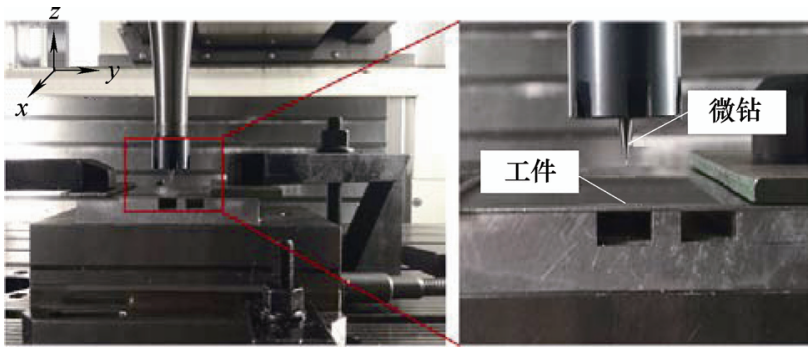

图 21 微细钻削试验装置

在主轴转速 $n$ 为 $14000 \mathrm{r} / \mathrm{min}$, 进给速度 $f$ 为 $28 \mathrm{~mm} / \mathrm{min}$, 钻削深度 $h$ 为 $1 \mathrm{~mm}$ 的条件下, 不同 螺旋槽型微细钻头的刀具缠屑情况和切屑形态如 图 22 和 23 所示。可以看出刀具 1 的缠屑最为严 重, 刀具 2 和刀具 3 没有明显的缠屑情况。对于 刀具 1 , 切屑基本为大螺距的带状长屑, 切屑沿螺 旋槽排出过程中容易缠绕在微细钻头钻体上, 导 致钻削过程中排屑困难。对于刀具 2 和刀具 3 , 产 生了多种形态的切屑, 主要有大螺距的带状切屑、 小螺距的螺卷形切屑以及断裂的单元屑, 相比刀 具 1 产生的带状长屑, 刀具 2 和刀具 3 所产生的 多为较小较碎的切屑, 带状长屑所占比例较低, 钻削过程中容易排屑, 不易发生因切屑阻塞造成 微细钻头折断的情况。

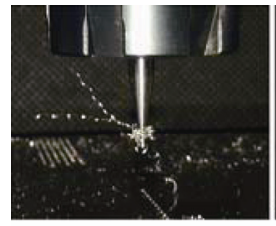

(a) 刀具 $1\left(\lambda=60^{\circ}\right)$

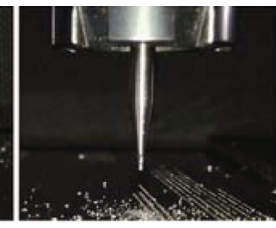

(b) 刀具 $2\left(\lambda=50^{\circ}\right)$

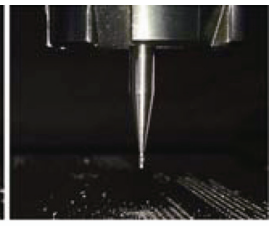

(c) 刀具 $3\left(\lambda=40^{\circ}\right)$
图 22 不同螺旋槽型微细钻头的刀具缠屑情况

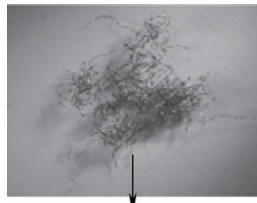

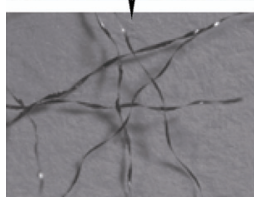

(a) 刀具 $1\left(\lambda=60^{\circ}\right)$

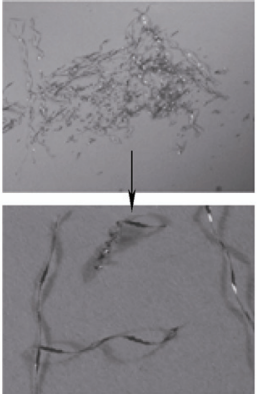

(b) 刀具 $2\left(\lambda=50^{\circ}\right)$

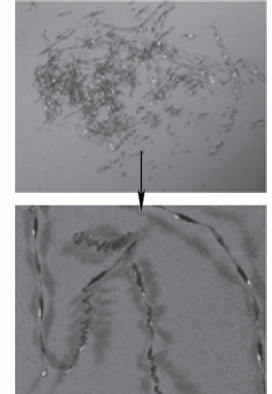

(c) 刀具 $3\left(\lambda=40^{\circ}\right)$
图 23 不同螺旋槽型微细钻头所产生的切屑形态

对于刀具 1 , 螺旋槽型由较大砂轮偏置角磨削 形成, 主切削刃为曲线化程度较高的凹形切削刃, 螺旋槽容屑空间小, 切屑侧卷和上卷程度较低, 形 成大螺距的带状长屑, 钻削过程中切屑容易缠绕在 刀体上, 增加了排屑难度, 并容易引起扭矩的增大, 造成微细钻头的折断。与刀具 1 相比, 刀具 2 和刀 具 3 的切削刃愈加接近直线刃, 螺旋槽的容屑空间 
依次增大, 切屑的卷曲程度剧烈, 形成的切屑有大 螺距带状形、小螺距螺卷形以及断屑的单元屑, 切 屑较短较碎, 钻削过程中容易排出。因此, 螺旋槽 结构对容屑与排屑能力具有重要影响。通过调整砂 轮形状和位置参数可以改变螺旋槽结构, 提高螺旋 槽的容屑与排屑能力。

\section{5 结论}

(1) 以微细钻头螺旋槽几何结构为研究对象, 基于包络原理建立螺旋槽的数学模型。基于 Matlab 仿真软件, 对螺旋槽横截面形状进行了数值仿真。 仿真结果表明随着砂轮边缘宽度和砂轮锥角的增 大, 径向前角没有变化, 但是螺旋槽宽度增大, 容 屑空间明显增大; 随着砂轮偏置角度的增大, 螺旋 槽径向前角明显增大, 螺旋槽宽度减小, 螺旋槽容 屑空间减小; 随着砂轮偏移距离的增大, 螺旋槽径 向前角轻微减小, 螺旋槽宽度明显增大, 螺旋槽容 屑空间增大。

（2）基于螺旋槽数学模型和砂轮几何、安装参 数, 计算各轴运动轨迹, 采用数控工具磨床进行微 细钻头螺旋槽的刃磨试验, 试验结果表明螺旋槽的 槽型、径向前角和槽宽与仿真具有较好的一致性, 验证了基于包络原理采用解析方法所建立的螺旋槽 横截面数学模型和仿真结果的正确性。采用所制备 的微细钻头进行钻削试验, 验证了螺旋槽结构对容 屑与排屑能力具有重要影响。

\section{参 考 文 献}

[1] ZHANG S, WANG X, LIANG Z, et al. Modeling and optimization of the flute profile of micro-drill[J]. International Journal of Advanced Manufacturing Technology, 2017, 92(5-8): 1-14.

[2] EHMANN K F, DEVRIES M F. Grinding wheel profile definition for the manufacture of drill flutes[J]. CIRP Annals-Manufacturing Technology, 1990, 39(1): 153-156.

[3] KANG Dechun, ARMAREGO E J A. COMPUTERÂ. Aided geometrical analysis of the fluting operation for twist drill design and production. ii. backward analysis, wheel profile, and simulation studies[J]. Machining Science and Technology, 2003, 7(2): 249-266.

[4] REN B Y, TANG Y Y, CHEN C K. The general geometrical models of the design and 2-axis $\mathrm{NC}$ machining of a helical end-mill with constant pitch[J]. Journal of Materials Processing Tech, 2001, 115(3): 265-270.
[5] CHEN W Y, CHANG P C, LIAW S D, et al. A study of design and manufacturing models for circular-arc ball-end milling cutters[J]. Journal of Materials Processing Technology, 2005, 161(3): 467-477.

[6] PHAM T T, KO S L. A manufacturing model of an end mill using a five-axis $\mathrm{CNC}$ grinding machine[J]. International Journal of Advanced Manufacturing Technology, 2010, 48(5-8): 461-472.

[7] ZHANG Wei, WANG Xianfeng, HE Fengbao, et al. A practical method of modelling and simulation for drill fluting[J]. International Journal of Machine Tools and Manufacture, 2006, 46(6): 667-672.

[8] BEJU L D, DAN P B, MUŢIU N C, et al. Modeling, simulation and manufacturing of drill flutes[J]. International Journal of Advanced Manufacturing Technology, 2016, 83(9-12): 2111-2127.

[9] XIAO S, WANG L, CHEN Z C, et al. A New and Accurate Mathematical Model for Computer Numerically

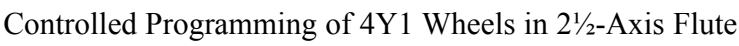
Grinding of Cylindrical End-Mills[J]. Journal of Manufacturing Science and Engineering, 2013， 135(4): 041008-041019.

[10] KARPUSCHEWSKI B, JANDECKA K, MOUREK D. Automatic search for wheel position in flute grinding of cutting tools[J]. CIRP Annals - Manufacturing Technology, 2011, 60(1): 347-350.

[11] TANG F, BAI J, WANG X. Practical and reliable carbide drill grinding methods based on a five-axis $\mathrm{CNC}$ grinder[J]. International Journal of Advanced Manufacturing Technology, 2014, 73(5-8): 659-667.

[12] REN L, WANG S, YI L, et al. An accurate method for five-axis flute grinding in cylindrical end-mills using standard 1V1/1A1 grinding wheels[J]. Precision Engineering, 2015, 43: 387-394.

[13] 张素燕, 梁志强, 王西涁, 等. 非共轴螺旋后刀面微钻 的五轴联动刃磨方法及其钻削性能研究 $[\mathrm{J}]$. 机械工程 学报, 2018, 54(19): 204-211.

ZHANG Suyan, LIANG Zhiqiang, WANG Xibin, et al. A 5-axis coordinated grinding method of non-coaxial helical flank micro-drill and its drilling performance research[J]. Journal of Mechanical Engineering, 2018, 54(19): 204-211.

[14] 何理论, 刘志兵, 王西涁, 等. 基于无瞬心包络的微细 铣刀螺旋槽刃磨分析 [J]. 机械工程学报, 2016, 52(19): 197-203.

HE Lilun, LIU Zhibing, WANG Xibin, et al. Grinding 
analysis for helical groove of micro mill based on non-instantaneous-pole envelope principle[J]. Journal of Mechanical Engineering, 2016，52(19): 197-203.

[15] ABELE E, FUJARA M. Simulation-based twist drill design and geometry optimization[J]. CIRP AnnalsManufacturing Technology, 2010, 59(1): 145-150.
作者简介: 梁志强(通信作者), 男, 1984 年出生, 博士, 副教授, 博士 生导师。主要研究方向为难加工材料切削磨削技术, 微细刀具设计与制 造技术。

E-mail: liangzhiqiang@bit.edu.cn

郭海新, 男, 1994 年出生, 硕士研究生。主要研究方向为微细钻削刀具 设计与制造技术。

E-mail: 18811318020@163.com

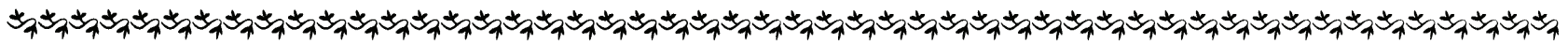

(上接第 184 页)

[29] WU Jiangtao, ZHAO Zeang, HAMEL C M, et al. Evolution of material properties during free radical photopolymerization[J]. Journal of the Mechanics and Physics of Solids, 2018, 112: 25-49.

[30] VITALE A, CABRAL J T. Frontal conversion and uniformity in $3 \mathrm{D}$ printing by photopolymerisation[J]. Materials, 2016, 9(9): 760.

[31] CABRAL J T, DOUGLAS J F. Propagating waves of network formation induced by light $[\mathrm{J}]$. Polymer, 2005, 46(12): 4230-4241.

[32] MU Xiaoming, SOWAN N, TUMBIC J A, et al. Photo-induced bending in a light-activated polymer laminated composite[J]. Soft Matter, 2015, 11(13): 2673-2682.

[33] FREUND L B. Substrate curvature due to thin film mismatch strain in the nonlinear deformation range[J]. Journal of the Mechanics and Physics of Solids, 2000, 48(6-7): 1159-1174.

[34] GE Qi, DUNN C K, QI H J, et al. Active origami by 4D printing[J]. Smart Materials and Structures, 2014, 23(9): 094007.

[35] SHARON E, EFRATI E. The mechanics of non-Euclidean plates[J]. Soft Matter, 2010，6(22): 5693-5704.

[36] SCHNEIDER C A, RASBAND W S, ELICEIRI K W. NIH Image to ImageJ: 25 years of image analysis[J]. Nature Methods，2012，9(7): 671.
[37] GLUGLA D J, ALIM M D, BYARS K D, et al. Rigid origami via optical programming and deferred self-folding of a two-stage photopolymer[J]. ACS Applied Materials \& Interfaces, 2016, 8(43): 29658-29667.

[38] CHESTER S A, DI LEO C V, ANAND L. A finite element implementation of a coupled diffusion-deformation theory for elastomeric gels[J]. International Journal of Solids and Structures, 2015，52: 1-18.

[39] MANSFIELD E H. The bending and stretching of plates[M]. Cambridge: Cambridge University Press, 2005.

[40] ABDULLAH A M, BRAUN P V, HSIA K J. Bifurcation of self-folded polygonal bilayers[J]. Applied Physics Letters, 2017, 111(10): 104101.

[41] ALBEN S, BALAKRISNAN B, SMELA E. Edge effects determine the direction of bilayer bending[J]. Nano Letters, 2011, 11(6): 2280-2285.

[42] ABDULlAH A M, BRAUN P V, HSIA K J. Programmable shape transformation of elastic spherical domes[J]. Soft Matter, 2016, 12(29): 6184-6195.

作者简介: 王金强, 男, 1992 年出生。主要研究方向为数字化设计制造, 自组装结构设计。

E-mail: wangjq@nuaa.edu.cn

戴宁(通信作者), 男, 1978 年出生, 博士, 副教授, 硕士研究生导师。 主要研究方向为增材设计制造技术、生物医学工程、逆向工程技术。 E-mail: dai_ning@nuaa.edu.cn 